\title{
Tumor-infiltrating T lymphocyte clonality predicts prognosis in human ovarian cancer
}

\author{
Takemasa Tsuji ${ }^{*}$, Kevin H Eng ${ }^{2}$, Sacha Gnjatic ${ }^{3}$, Junko Matsuzaki ${ }^{1}$, Rachel Brightwell ${ }^{4}$, Anthony Miliotto ${ }^{1}$, \\ Ryan Emerson ${ }^{5}$, Cindy Desmarais ${ }^{5}$, Erika Lindsley ${ }^{5}$, Julie Rubinstein ${ }^{5}$, Jianmin Wang ${ }^{2}$, Song Liư ${ }^{2}$, Harlan Robins ${ }^{5}$, \\ Kunle Odunsi ${ }^{1,4}$
}

From Society for Immunotherapy of Cancer 28th Annual Meeting

National Harbor, MD, USA. 8-10 November 2013

\section{Background}

The prognostic significance of the number of tumorinfiltrating $\mathrm{T}$ cells has been demonstrated for many tumor types. In contrast, the significance of the tumorinfiltrating $\mathrm{T}$ cell clonality, which reflects the preferential infiltration or expansion of $\mathrm{T}$ cell clones in the tumor microenvironment, has not been clear because of the technical hurdles required for evaluating each $\mathrm{T}$ cell clone in the tumor. In order to delineate the complexity of $\mathrm{T}$ cell responses and define correlates of a protective immunity, we applied a recently developed deep $\mathrm{T}$ cell receptor (TCR)-sequencing technology (immunoSEQ) to paired frozen tumor tissues and peripheral blood mononuclear cells from 99 ovarian cancer patients.

\section{Methods}

Rearranged $\beta$ TCR chain DNA sequences were sequenced using immunoSEQ technology. Frequency of each T cell clone was obtained from the copy number of the sequence. $T$ cell clonality of the specimens was calculated from entropy of TCR sequences. Spontaneous immune responses against tumor-associated antigens (NY-ESO-1, MAGE-A1, MAGE-A3 and p53) were evaluated by measuring serum antibodies by ELISA.

\section{Results}

Approximately $3( \pm 3) \times 10^{6}$ and $4( \pm 2) \times 10^{6}$ full-length TCR beta chain sequences were obtained corresponding to the detection limit for $\mathrm{T}$ cell frequency at $3 \times 10^{-7}$ and $2 \times 10^{-7}$ for tumor and blood samples, respectively. In patients who had spontaneous antibodies against a panel of tumor-associated antigens, more clonal $\mathrm{T}$ cell infiltration was associated with longer progression-free survival. In sharp contrast, clonal infiltration was a worse prognostic factor in patients without detectable humoral immune responses against surrogate tumor antigens. From sequence based analyses, we found a set of shared TCR sequences among patients.

\section{Conclusion}

Deep TCR sequencing using immunoSEQ technology is a powerful tool to characterize tumor-infiltrating $\mathrm{T}$ cell clonality using frozen tumor tissues. Our analyses indicate that evaluation for spontaneous anti-tumor immune responses is required to correctly understand the prognostic significance of tumor-infiltrating $\mathrm{T}$ cells.

\section{Authors' details}

${ }^{1}$ Center for Immunotherapy, Roswell Park Cancer Institute, Buffalo, NY, USA ${ }^{2}$ Biostatistics and Bioinformatics, Roswell Park Cancer Institute, Buffalo, NY, USA. ${ }^{3}$ Tisch Cancer Institute, Icahn School of Medicine at Mount Sinai, New York, NY, USA. ${ }^{4}$ Gynecologic Oncology, Roswell Park Cancer Institute, Buffalo, NY, USA. ${ }^{5}$ Adaptive Biotechnologies, Seattle, WA, USA.

Published: 7 November 2013

doi:10.1186/2051-1426-1-S1-P64

Cite this article as: Tsuji et al:: Tumor-infiltrating $\mathrm{T}$ lymphocyte clonality predicts prognosis in human ovarian cancer. Journal for ImmunoTherapy of Cancer 2013 1(Suppl 1):P64. 